\title{
Explaining variability in ciclosporin exposure in adult kidney transplant recipients
}

\author{
Rogier R. Press • Bart A. Ploeger • Jan den Hartigh - T. van der Straaten • \\ Hans van Pelt • Meindert Danhof • Hans de Fijter • Henk-Jan Guchelaar
}

Received: 19 June 2009 / Accepted: 28 February 2010 /Published online: 31 March 2010

(C) The Author(s) 2010. This article is published with open access at Springerlink.com

\begin{abstract}
Purpose Optimal ciclosporin A (CsA) exposure in kidney transplant recipients is difficult to attain because of variability in CsA pharmacokinetics. A better understanding of the variability in CsA exposure could be a good means of individualizing therapy. Specifically, genetic variability in genes involved in CsA metabolism could explain exposure differences. Therefore, this study is aimed at identifying a relationship between genetic polymorphisms and the vari-
\end{abstract}

R. R. Press $(\bowtie) \cdot$ J. den Hartigh $\cdot$ T. van der Straaten •

H.-J. Guchelaar

Department of Clinical Pharmacy and Toxicology,

Leiden University Medical Center,

Albinusdreef 2,

2333 ZA Leiden, The Netherlands

e-mail: r.r.press@lumc.nl

R. R. Press $\cdot$ H. de Fijter

Department of Nephrology, Leiden University Medical Center,

Albinusdreef 2,

2333 ZA Leiden, The Netherlands

H. van Pelt

Department of Clinical Chemistry,

Leiden University Medical Center,

Albinusdreef 2,

2333 ZA Leiden, The Netherlands

B. A. Ploeger · M. Danhof

Leiden Amsterdam Center for Drug Research (LACDR),

Einsteinweg 55,

2333 CC Leiden, The Netherlands

B. A. Ploeger - M. Danhof

Leiden Experts on Advanced

Pharmacokinetics and Pharmacodynamics

(LAP\&P Consultants BV),

Archimedesweg 31,

2333 CM Leiden, The Netherlands ability in CsA exposure, while accounting for non-genetic sources of variability.

Methods De novo kidney transplant patients $(n=33)$ were treated with CsA for 1 year and extensive blood sampling was performed on multiple occasions throughout the year. The effects of the non-genetic covariates hematocrit, serum albumin concentration, cholesterol, demographics (i.e., body weight), CsA dose interval, prednisolone dose and genetic polymorphisms in genes encoding ABCB1, CYP3A4, CYP3A5, and PXR on CsA pharmacokinetics were studied using non-linear mixed effect modeling.

Results The pharmacokinetics of CsA were described by a two-compartment disposition model with delayed absorption. Body weight was identified as the most important covariate and explained $35 \%$ of the random inter-individual variability in CsA clearance. Moreover, concurrent prednisolone use at a dosage of $20 \mathrm{mg} /$ day or higher was associated with a $22 \%$ higher clearance of CsA, hence lower CsA exposure. In contrast, no considerable genotype effects (i.e., greater than $30-50 \%$ ) on CsA clearance were found for the selected genes. Conclusions It appears that the selected genetic markers explain variability in CsA exposure insufficiently to be of clinical relevance. Therefore, therapeutic drug monitoring is still required to optimize CsA exposure after administration of individualized doses based on body weight and, as this study suggests, co-administration of prednisolone.

Keywords Ciclosporin A - Therapeutic drug monitoring · Population pharmacokinetics $\cdot$ Pharmacogenetics

\section{Introduction}

The trend toward lowering the exposure of the calcineurin inhibitor ciclosporin (CsA) is widely propagated [1] as CsA 
exposure, in terms of drug blood concentrations, relates to the clinical endpoints rejection and toxicity [2]. Since therapy with CsA is characterized by considerable interand intra-individual variability [3] in its pharmacokinetics (PK), it is difficult to remain within the therapeutic window. Therefore, to optimize therapy in clinical practice, therapeutic drug monitoring (TDM), aimed at individualized CsA dosing is common practice $[2,4,5]$. As a next step, to further optimize CsA therapy, insight into the sources of this variability in CsA exposure is necessary.

It has been demonstrated that the observed variability in CsA PK originates from non-genetic biological and lifestyle-related factors, including age, body size, gender, food intake [3], serum albumin concentration, hematocrit, lipoproteins (HDL, LDL) [6, 7], and co-administration of interacting drugs [3, 8-11]. Yet, even when these factors are taken into account, a considerable part of the variability remains unexplained, which could potentially be attributed to genetic differences between patients. Insight into this relationship could aid in optimizing CsA exposure early after transplantation. Indeed, CsA disposition is characterized by extensive metabolism by cytochrome P450 (CYP) enzymes $[12,13]$. Moreover, CsA is a substrate for the efflux pump P-glycoprotein (ABCB1) [12, 13]. Recently, the pregnane $\mathrm{X}$ receptor (PXR) was reported to be the key nuclear receptor regulating expression of cytochrome enzymes and certain transport proteins and mediating their induction [14-16]. To date, no studies have been published relating CsA PK parameters to polymorphisms in PXR. Several investigators have studied the role of genetic variants in genes encoding for the drug metabolizing enzymes CYP3A4, CYP3A5, and the multidrug resistance transporter $\mathrm{ABCB} 1[13,17]$. These studies show conflicting results with regard to the contribution of single nucleotide polymorphisms (SNPs) in $A B C B 1$ C1236T, T3435C, G2677A and T129C, CYP3A4*1B, and CYP3A5*1 [18-22].

A limitation of many studies is that often other sources of variability, which can mask the actual relationship between genetic factors and CsA exposure, have not been taken into account. This is especially the case because associations between SNPs in these enzymes are mainly related to dose-corrected trough concentrations, which are not a very sensitive measure of variability in exposure. An integrated analysis on the basis of full concentration vs time profiles, accounting for the observed variability in CsA PK by including a wide range of covariates, is the approach for identifying any relationship between CsA exposure and genetic polymorphisms in genes encoding CYP3A4, CYP3A5, P-glycoprotein or the pregnane $\mathrm{X}$ receptor. Therefore, in this study, a population analysis of CsA PK was performed, aimed at a comprehensive exploration of the determinants for individualizing the CsA dose in kidney transplant recipients.

\section{Materials and methods}

Patients and therapy

De novo kidney transplant patients $(n=33)$ aged between 18 and 70 years were followed for 1 year after transplantation (Table 1). Recipient, donor, and transplant characteristics as well as outcome parameters (acute rejection rate, patient and graft survival, and renal function) are summarized in Table 1. Only recipients of a first kidney graft from a deceased or living (non-HLA-identical) donor were included. From each patient written informed consent was obtained. The study was performed in accordance with the Declaration of Helsinki and was approved by the Medical Ethics Committee of the Leiden University Medical Center.

Patients received quadruple immunosuppression consisting of induction therapy with basiliximab on the day of transplantation and on day 4 , a fixed dose $(1,000 \mathrm{mg}$ twice daily) of mycophenolate mofetil (MMF), prednisolone (50 $\mathrm{mg}$ twice daily on the day of transplantation, but rapidly tapered toward $10 \mathrm{mg}$ once daily at day 22) and CsA $\left(\mathrm{Neoral}^{\circledR}\right)$. Patients were randomized to receive a CsA daily dose of $8 \mathrm{mg} / \mathrm{kg} /$ day in either a once or twice daily regimen (Table 1). TDM for twice daily CsA was aimed at a target AUC of $5,400 \mu \mathrm{g}^{*} \mathrm{~h} / \mathrm{L}$ in the first 6 weeks and at $3,250 \mu \mathrm{g} * \mathrm{~h} / \mathrm{L}$ after this period. Likewise, for the once daily regimen these values were $10,800 \mu \mathrm{g} * \mathrm{~h} / \mathrm{L}$ and $6,500 \mu \mathrm{g}^{*} \mathrm{~h} / \mathrm{L}$ respectively.

\section{Therapeutic drug monitoring}

Therapeutic drug monitoring (TDM) was performed on the basis of a limited sampling strategy (blood concentration at $\mathrm{t}=0,2$, and $3 \mathrm{~h}$ ) and the Bayesian estimation of the $\mathrm{AUC}_{[0-}$ $12 \mathrm{~h}$ ] using MW/Pharm version 3.5 (Mediware, Groningen, The Netherlands), as described previously [4]. Routine TDM samples were taken during the mornings of weeks 4 , $8,10,17,21$, and 39 after transplantation. In addition, PK was densely sampled in weeks $2,6,12,26$, and 52 with samples at $\mathrm{t}=0,1,2,3,4,6$, and $24 \mathrm{~h}$. A majority of patients (22) was sampled on each of the eleven study occasions, while 6 patients were sampled on ten occasions and 2 patients on twelve occasions. The remaining 3 patients were sampled less frequently (ranging from three to six occasions). Furthermore, at every TDM visit CsA dosage information was recorded, which consisted of the actual time of dosing (that morning) and the time of dosing the evening before, as well as the amount and dose interval. As patients had to use the same dose for at least 3 days to reach a steady state, the start of therapy or the last date of dose change was recorded.

Ciclosporin A concentrations were determined in whole blood by fluorescence polarization immunoassay 
Table 1 Clinical characteristics

$D M$ diabetes mellitus, $D D$ deceased donor, $L R D$ living related donor, $L U R D$ living unrelated donor, $H L A$ human leukocyte antigen, $A T G$ anti-thymocyte globulin, $S D$ standard deviation

\begin{tabular}{|c|c|c|c|}
\hline Variable & $\begin{array}{l}\text { Once daily } \\
(N=17)\end{array}$ & $\begin{array}{l}\text { Twice daily } \\
(N=16)\end{array}$ & $p$ value \\
\hline \multicolumn{4}{|l|}{ Recipient characteristics } \\
\hline Age $($ mean $\pm \mathrm{SD})$ & $43.8 \pm 14.5$ & $48.9 \pm 10.5$ & 0.79 \\
\hline Male sex $(n)$ & 15 & 11 & 0.23 \\
\hline Caucasian $(n)$ & 14 & 12 & 0.69 \\
\hline Native kidney disease & & & 0.29 \\
\hline Pyelonephritis & 0 & 2 & \\
\hline Glomerulonephritis & 6 & 6 & \\
\hline Hereditary/congenital & 2 & 4 & \\
\hline Hypertension & 4 & 0 & \\
\hline $\mathrm{DM}$ & 1 & 2 & \\
\hline Other & 2 & 1 & \\
\hline Unknown & 2 & 1 & \\
\hline \multicolumn{4}{|l|}{ Donor characteristics } \\
\hline Age $($ mean $\pm \mathrm{SD})$ & $44.9 \pm 16.7$ & $43.1 \pm 12.6$ & 0.72 \\
\hline Male sex $(n)$ & 7 & 6 & 0.83 \\
\hline DD heart beating & 7 & 9 & 0.49 \\
\hline DD non-heart beating & 6 & 1 & 0.09 \\
\hline LRD & 2 & 4 & 0.40 \\
\hline LURD & 2 & 2 & 0.95 \\
\hline \multicolumn{4}{|l|}{ Transplant characteristics } \\
\hline HLA mismatch mean \pm SD & $2.00 \pm 1.50$ & $2.38 \pm 1.82$ & \\
\hline Class I & $1.47 \pm 1.23$ & $1.63 \pm 1.36$ & 0.79 \\
\hline Class II & $0.53 \pm 0.51$ & $0.75 \pm 0.68$ & 0.40 \\
\hline Cold ischemia time (h) DD only & $19.9 \pm 4.3$ & $23.0 \pm 8.4$ & 0.25 \\
\hline Acute rejection 6 months $(n)$ & 4 & 2 & 0.40 \\
\hline Need for ATG $(n)$ & 3 & 1 & 0.33 \\
\hline \multicolumn{4}{|l|}{ Patient survival $(n)$} \\
\hline 1 year & 17 & 16 & \\
\hline 2 years & 17 & 16 & \\
\hline Death-censored graft survival $(n)$ & & & 0.60 \\
\hline 1 year & 16 & 16 & \\
\hline 2 years & 16 & 16 & \\
\hline Nankivell clearance (mean \pm SD) & & & 0.44 \\
\hline Week 2 & $45 \pm 23$ & $48 \pm 21$ & \\
\hline Week 6 & $62 \pm 17$ & $61 \pm 15$ & \\
\hline Month 3 & $64 \pm 14$ & $61 \pm 13$ & \\
\hline Month 6 & $68 \pm 16$ & $64 \pm 10$ & \\
\hline Month 9 & $68 \pm 15$ & $63 \pm 11$ & \\
\hline Year 1 & $69 \pm 17$ & $64 \pm 12$ & \\
\hline Year 2 & $64 \pm 16$ & $60 \pm 11$ & \\
\hline
\end{tabular}

Genotyping assays

The DNA was isolated from EDTA blood. Primers and probes used in the Taqman-based genotyping assays, as well as primers and sequences used in the pyrosequence assays are listed in Table 2. ABCB1 C1236T (rs1128503), T3435C (rs1045642), and G2677T (rs2032582) were determined with $800 \mu \mathrm{g} / \mathrm{L}$. 
Table 2 Primers and probes for TaqMan and pyrosequence analysis

\begin{tabular}{|c|c|c|c|}
\hline SNP & Target & Sequence $5^{\prime}-3$ & Modification $^{\mathrm{a}}$ \\
\hline \multirow[t]{4}{*}{$A B C B 1 C 1236 \mathrm{~T}$} & PCR-f & CACCGTCTGCCCACTCT & \\
\hline & PCR-r & GTGTCTGTGAATTGCCTTGAAGTTT & \\
\hline & Probe- $\mathrm{T}$ & TTCAGGTTCAGACCCTT & VIC \\
\hline & Probe-C & CAGGTTCAGGCCCTT & FAM \\
\hline \multirow[t]{4}{*}{$A B C B 1 \mathrm{G} 2677 \mathrm{~T}$} & PCR-f & CTTAGAGCATAGTAAGCAGTAGGGAGT & \\
\hline & PCR-r & GAAATGAAAATGTTGTCTGGACAAGCA & \\
\hline & Probe-G & TTCCCAGCACCTTC & VIC \\
\hline & Probe-T & TTCCCAGAACCTTC & FAM \\
\hline \multirow[t]{5}{*}{$A B C B 1 \mathrm{~T} 3435 \mathrm{C}$} & PCR-f & ATGTATGTTGGCCTCCTTTGCT & \\
\hline & PCR-r & GCCGGGTGGTGTCACA & \\
\hline & Probe- $\mathrm{T}$ & СССТСАСААТСТСТ & VIC \\
\hline & Probe-C & CCCTCACGATCTCT & FAM \\
\hline & Pyrosequence & & \\
\hline \multirow[t]{4}{*}{$A B C B 1 \mathrm{~T}-129 \mathrm{C}$} & PCR-f & TCGAAGTTTTTATCCCA & Biotine \\
\hline & PCR-r & ССТCСТGGAAATTCAACCTGTT & \\
\hline & Sequence primer & TACTCCGACTTTAGTGGAAAGACC & \\
\hline & Target sequence & CTG/ACTCGAATGAG & \\
\hline \multirow[t]{4}{*}{$C Y P 3 A 5 * 3$} & PCR-f & CTGCCTTCAATTTTTCACT & \\
\hline & PCR-r & TATGTTATGTAATCCATACCCC & Biotine \\
\hline & Sequence primer & AGAGCTCTTTTGTCTTTCA & \\
\hline & Target sequence & A/GTATCTC & \\
\hline \multirow[t]{4}{*}{ CYP $3 A 4 * 1 \mathrm{~B}$} & PCR-f & CAGCCATAGAGACAAGGGC & \\
\hline & PCR-r & GAAGAGGCTTCTCCACCTT & Biotine \\
\hline & Sequence primer & CCATAGAGACAAGGGCA & \\
\hline & Target sequence & A/GGAGAGAGG & \\
\hline \multirow[t]{4}{*}{ CYP $3 A 5 * 6$} & PCR-f & TCTTTGGGGCCTACAGCATG & \\
\hline & PCR-r & AAAGAAATAATAGCCCACATACTTATTGAGAG & Biotine \\
\hline & Sequence primer & AGAAACCAAATTTTAGGAA & \\
\hline & Target sequence & CTTC/TTTAG & \\
\hline \multirow[t]{4}{*}{$P X R \mathrm{C}-25385 \mathrm{~T}$} & PCR-f & GTGGTCATTTTTTGGCAATCCC & \\
\hline & PCR-r & AGCCTCTGGCAACAGTAAAGCA & Biotine \\
\hline & Sequence primer & TTGGCAATCCCAGGT & \\
\hline & Target sequence & TC/TTCTTTTCTACCTGTT & \\
\hline \multirow[t]{4}{*}{$P X R$ A-24381C } & PCR-f & AGTGGGAATCTCGGCCTCA & \\
\hline & PCR-r & CTGGGGTCCACTTTGAACAATC & Biotine \\
\hline & Sequence primer & GCTAATACTCCTGTCCTGAA & \\
\hline & Target sequence & A/CAAGGCAGCGGCTCCTTG & \\
\hline \multirow[t]{4}{*}{$P X R \mathrm{G}-24113 \mathrm{~A}$} & PCR-f & GAATCATGTTGGCCTTGCTGC & \\
\hline & PCR-r & GCATCAGTAATGGGGCTCAAC & Biotine \\
\hline & Sequence primer & TCTCCTCATTTCTAGGGT & \\
\hline & Target sequence & C/TCACCCTAG & \\
\hline \multirow[t]{4}{*}{$P X R \mathrm{~A}+252 \mathrm{G}$} & PCR-f & TGCAAGGGCTTTTTCAGGTAGAGT & \\
\hline & PCR-r & TGAACCTGGGGGATAGGTCAAG & Biotine \\
\hline & Sequence primer & ACTGACCCACTGGGTAA & \\
\hline & Target sequence & CA/GTCTCAGGGC & \\
\hline \multirow[t]{4}{*}{$P X R \mathrm{~A}+7635 \mathrm{G}$} & PCR-f & AGCCATCCTCCСТCTTC & Biotine \\
\hline & PCR-r & CAGCAGCCATCCCATAATC & \\
\hline & Sequence primer & CATAATCCAGAAGTTGGG & \\
\hline & Target sequence & GGC/TGAGAGGAA & \\
\hline
\end{tabular}

$f$ forward orientated, $r$ reverse orientated, $A B C$ ATP-binding cassette, $C Y P$ cytochrome $\mathrm{P} 450, P X R$ pregnane $\mathrm{X}$ receptor, $P C R$ polymerase chain reaction

${ }^{\mathrm{a}} \mathrm{VIC}$ and FAM are fluorescent dyes, biotine is necessary to obtain single-stranded DNA 
TaqMan 7500 (Applied Biosystems, Nieuwerkerk aan den IJssel, The Netherlands) with custom designed assays, according to the manufacturer's protocol. $A B C B 1 \mathrm{~T}-129 \mathrm{C}$ (rs3213619), CYP3A5*3/*6 (rs776746/rs10264272), CYP $3 A 4^{*} 1 \mathrm{~B}$ (rs2740574), and NR1I2 or PXR SNPs C25385T (rs3814055), A-24381C (rs1523127), G-24113A (rs2276706), A+252G (rs1464603), and $A+7635 G$ (rs6785049) $[14,15]$ were determined with Pyrosequencer 96MA (Isogen, IJsselstein, The Netherlands). In short, PCR reactions contained $10 \mathrm{ng}$ of DNA, and 5 pmol of each PCR primer (Table 2) in a total volume of $12 \mu \mathrm{L}$. Cycle conditions were: initial denaturation for $15 \mathrm{~min}$ at $95^{\circ} \mathrm{C}, 35$ cycles of $95^{\circ} \mathrm{C}-55^{\circ} \mathrm{C}-72^{\circ} \mathrm{C}$ each for $30 \mathrm{~s}$, ending with $10 \mathrm{~min}$ at $72^{\circ} \mathrm{C}$. The pyrosequence reactions were performed according to the manufacturer's protocol. The sequence used for analysis and the calculated dispensation order for each SNP are listed in Table 2. Note that the lower case nucleotides in the dispensation sequence are negative controls, which are not incorporated into the target DNA and consequently should not appear in the pyrogram. As quality control, $5 \%$ of samples were genotyped in duplicate. In addition, negative controls (water) were used. The allele frequencies were found to be in HardyWeinberg equilibrium. Table 3 presents the genotype distribution in the overall genotyped population. The haplotype analysis for ABCB1 SNPs that are in linkage disequilibrium was performed using gPLINK with haplotypes set with a certainty greater than 0.97 .

Population pharmacokinetic analysis

The PK of CsA was analyzed by non-linear mixed effects modeling. Mixed effects models consist of a structural model, describing the relationship between dose and concentration in terms of structural PK parameters (i.e., $\mathrm{CL}, \mathrm{V})$, and a stochastic model, describing the random variability in the structural model parameters. The random effects are the expression of inter-individual and interoccasion variability. Inter-individual variability describes the random variability of structural parameters within the population, whereas inter-occasion variability describes the variability of an individual parameter value from one occasion to another. The second level of stochastic effects, $\sigma^{2}$, describes the variability of the difference between observed and predicted responses. This residual error includes, among other factors, model mis-specification, intra-individual variability, and measurement error. In the mixed effects modeling approach, structural and stochastic parameters are simultaneously estimated by fitting the model to the data. In this respect the following parameters were estimated: PK parameters, variance and covariance $\left(\omega^{2}\right)$ of each individual specific parameter value $(\eta)$ and variance $\left(\sigma^{2}\right)$ of the residual error. As a result, individual post hoc estimates of parameters associated with interindividual and inter-occasion variability could be obtained.

\section{Structural model}

The PK of CsA was fitted to linear compartmental models. The value for the oral bioavailability was fixed at $50 \%$, as previously described $[3,23]$ and used in the clinically applied TDM model [4].

\section{Random effects}

Inter-individual variability (IIV) and inter-occasion variability (IOV) were described assuming a log normal distribution with the following equation:

Table 3 Genotype distribution in the study population $(n=33)$. The number of individuals carrying a certain genotype are presented

\begin{tabular}{|c|c|c|c|c|}
\hline SNP & Frequency & & & \\
\hline ABCB1 T3435C & $\mathrm{T} / \mathrm{T} 9$ & $\mathrm{C} / \mathrm{T} 12$ & $\mathrm{C} / \mathrm{C} 10$ & $2 \mathrm{UG}$ \\
\hline ABCB1 G2677T & $\mathrm{G} / \mathrm{G} 10$ & G/T 13 & $\mathrm{~T} / \mathrm{T} 7$ & $3 \mathrm{UG}$ \\
\hline ABCB1 C1236T & $\mathrm{C} / \mathrm{C} 10$ & C/T 14 & $\mathrm{~T} / \mathrm{T} 7$ & $2 \mathrm{UG}$ \\
\hline ABCB1 T-129C & T/T 29 & $\mathrm{C} / \mathrm{T} 2$ & $\mathrm{C} / \mathrm{C} 0$ & $2 \mathrm{UG}$ \\
\hline CYP3A4*1B & A/A 27 & G/A 3 & $\mathrm{G} / \mathrm{G} 1$ & $2 \mathrm{UG}$ \\
\hline CYP3A $5 * 3$ & G/G 25 & $\mathrm{~A} / \mathrm{G} 4$ & $\mathrm{~A} / \mathrm{A} 2$ & $2 \mathrm{UG}$ \\
\hline CYP3A $5 * 6$ & C/C 28 & $\mathrm{C} / \mathrm{T} 3$ & $\mathrm{~T} / \mathrm{T} 0$ & $2 \mathrm{UG}$ \\
\hline PXR C-25385 T & $\mathrm{C} / \mathrm{C} 7$ & $\mathrm{C} / \mathrm{T} 16$ & $\mathrm{~T} / \mathrm{T} 8$ & $2 \mathrm{UG}$ \\
\hline PXR A-24381C & $\mathrm{A} / \mathrm{A} 6$ & $\mathrm{~A} / \mathrm{C} 15$ & $\mathrm{C} / \mathrm{C} 10$ & $2 \mathrm{UG}$ \\
\hline PXR G-24113A & $\mathrm{A} / \mathrm{A} 8$ & G/A 16 & $\mathrm{G} / \mathrm{G} 7$ & $2 \mathrm{UG}$ \\
\hline PXR A+252G & $\mathrm{A} / \mathrm{A} 10$ & G/A 15 & G/G 6 & $2 \mathrm{UG}$ \\
\hline PXR A+7635G & $\mathrm{A} / \mathrm{A} 6$ & G/A 19 & $\mathrm{G} / \mathrm{G} 6$ & $2 \mathrm{UG}$ \\
\hline
\end{tabular}

Frequency determined in successfully genotyped individuals

SNP single nucleotide polymorphism, $A B C$ ATP-binding cassette, $C Y P$ cytochrome $\mathrm{P} 450, P X R$ pregnane $\mathrm{X}$ receptor; $U G$ unsuccessfully genotyped ( 2 individuals in particular) 
$P K j=T V P K \times e^{\eta j P K}$ in which $P K j$ is the PK parameter in the $j$ th individual and $\eta j_{P K}$ is the difference between the individual specific parameter and the population value. TVPK is the population value of the PK parameter and the difference in the logarithm between the individual value of the subject $j$ and the population mean $\left(\eta j_{\mathrm{PK}}\right)$ is normally distributed with a mean of zero and variance $\omega_{\mathrm{PK}}^{2}$. The residual error was assumed to be additive to the predicted concentration after $\log$ transformation: $\log (C i j)=$ $\log ($ Cpredij $)+\varepsilon i j$ in which $c i j$ is the Ith observation for the $j$ th individual, Cpredij is the concentration of CsA in the blood predicted by the PK model, and $\varepsilon i j$ (the difference between $C i j$ and $C p r e d i j$ ) is a normally distributed variable with a mean of zero and variance $\sigma^{2}$.

\section{Covariate analysis}

The non-genetic biological and life-style covariates hematocrit, serum albumin concentration, prednisolone daily dose, CsA dose regimen, cholesterol (LDL, HDL), the demographic factors body weight, age, sex, and body surface area (BSA), and the genetic markers for $A B C B 1$ (T3435C, G2677T, C1236T, T-129C), CYP3A4 (*1B), CYP3A5 $\left(* 3,{ }^{*} 6\right)$, and NR1I2/PXR (C-25385T, A-24381C, G-24113A, A+252G, A+7635G) were selected on the basis of their known or theoretical relationships with CsA PK. Covariates with a clear visual relationship between the random effects in the model without covariates (base model) and the covariate values were formally tested with the model. When the relationship was described allometrically (i.e., in a body weight-adjusted manner), it was done in the form $P K=T V P K x(B W / \text { mean } B W)^{y}$, where $B W$ is the individual body weight value, mean $B W$ is the body weight population mean, and $y$ is the allometric exponent, typically with a value of 0.75 for clearance and 1 for volume of distribution [24]. Subsequently, the selected covariate relationships were evaluated by a forward inclusion and a backward deletion procedure [25]. Including a covariate effect should result in a reduction in the identified random variability and an improvement in the model fit.

\section{Computation}

Non-linear mixed effects modeling (NONMEM, version VI release 1.2, Icon Development Solutions, Ellicott City, MD, USA) [26] was used for modeling CsA PK. Modeling results were analyzed using the statistical software package S-Plus ${ }^{\circledR}$ for Windows (version 6.2 Professional; Insightful, Seattle, WA, USA). A convergence criterion of 3 significant digits in the parameter estimates was used. For model comparisons, the minimum value obtained of the objective function (MVOF), defined as minus twice the log likelihood, was used. First-order conditional estimation (FOCE) with interaction was used throughout the modeling process. The modeling process was guided by statistical and visual checks (i.e., diagnostic "goodness of fit" plots). A model parameter or a covariate was retained in the model when including this parameter in the model resulted in a decrease of 6.63 points ( $\chi^{2}$ distribution, 1 degree of freedom, $p=$ $0.01)$ in the minimum value of the objective function $(\triangle \mathrm{MVOF} \geq 6.63$ ) or vice versa with backward deletion from the model. This conservative $p$ value (Type I error) was selected, since it is known that the NONMEM FOCE method produces only an approximation to the maximum likelihood assumptions and that the null hypothesis will be rejected more frequently than the nominal Type I error value [27].

\section{Visual predictive check}

The model prediction was evaluated using a visual predictive check (VPC), which evaluates whether the identified model would be able to predict the observed variability for $80 \%$ of the population in the PK data that were used for model identification [28]. Therefore, the PK of each individual using its individual specific dosing history and covariate values was simulated using the individual specific values for dose and covariates. In a Monte Carlo simulation, 100 datasets were simulated by drawing random samples for the PK parameters from the identified distributions for inter-individual variability, interoccasion variability, and residual variability. The distribution (median and 10th and 90th percentiles) of the simulated concentration-time courses was compared with the distribution of the observed values in the original dataset. Differences and overlap of the simulated and original distributions indicate the accuracy of the identified model.

\section{Bootstrap}

A bootstrap analysis was performed to assess the precision of the PK parameter estimates. The observed dataset was re-sampled with replacement in order to generate a new dataset with the same size and population characteristics, such as the number of patients per genotype, as the original set. This procedure was repeated 500 times to generate a distribution of the PK parameters with a mean and coefficient of variation as well as the median and 2.5th and 97.5 th percentiles.

\section{Results}

Ciclosporin A PK is characterized by variable peak concentrations in the first $3 \mathrm{~h}$ after administration. The 
variability in the delayed absorption of CsA could best be described with a transit compartment, using a first-order rate constant describing the transfer from the dose compartment into the transit compartment and subsequently into the central compartment (Fig. 1). Distribution and elimination of CsA could be adequately described by a twocompartment model with first-order elimination (Fig. 1). Random effects for the inter-individual variability were estimated for clearance; the volume of the central compartment and the absorption rate were constant.

The value of the absorption rate constant varied from 0.5 to $3.2 \mathrm{~h}^{-1}$. The first-order transit rate constant is set to be equal to the absorption rate constant; thus, the transit time can be calculated with $1 / \mathrm{k}_{\mathrm{a}} *(n+1)$, where $n$ is the number of transit compartments [29]. The transit time or lag time was typically $1 \mathrm{~h}$ (range 0.6 to $4 \mathrm{~h}$ ). A concomitant prednisolone dose of $20 \mathrm{mg} /$ day or higher was related to a $55 \%$ lower $\mathrm{CsA}$ absorption rate $(\Delta \mathrm{MVOF}=+233$ points, deletion from the final model, Table 4).

The range in apparent clearance $(\mathrm{CL} / \mathrm{F})$ in the population was $13-64 \mathrm{~L} / \mathrm{h}$, with a median of $32 \mathrm{~L} / \mathrm{h}$. The relationships among body weight, CsA clearance, and the central volume of distribution were described allometrically and the parameters were scaled to the median body weight, i.e., $\mathrm{CL}=15 \times\left(\right.$ body weight/76) ${ }^{0.75}$. The body weight range in the population was 49 to $140 \mathrm{~kg}$ with a median of $76 \mathrm{~kg}$. Incorporating body weight into the model explained $9 \%$ of the inter-individual variability in $\mathrm{CL} / \mathrm{F}$ (decrease from 26 to $17 \%$, while it accounted for $8 \%$ (decrease from 43 to $35 \%$ ) of the inter-individual variability in the volume of the central compartment $(\Delta \mathrm{MVOF}=+17$ when deleting the effect of body weight on both parameters from the final model). This means that, relative to the observed variability, $35 \%$ and $19 \%$ of the inter-individual variability in these parameters are explained by the covariate body weight respectively.

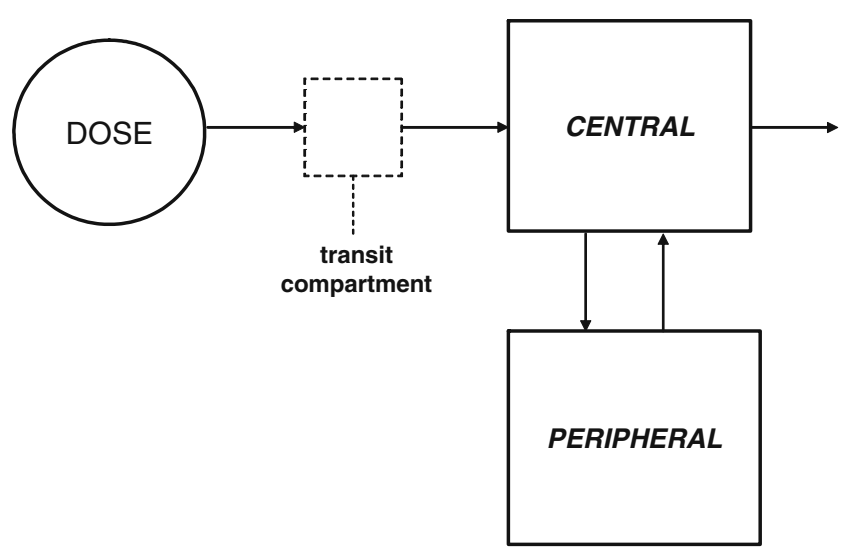

Fig. 1 Linear two-compartment model with first-order absorption and elimination and a transit compartment in order to describe the variability in the absorption phase
Inter-occasion variability was estimated for the fixed bioavailability term (Table 4 ) and not for the clearance term because of a better model fit. The model clearly improved when accounting for a $22 \%$ lower bioavailability if a prednisolone dose of $20 \mathrm{mg} /$ day or higher was coadministered $(\triangle \mathrm{MVOF}=+51$, deletion from final model). This accounted for $20 \%$ of the inter-occasion variability as the variability value decreased to $14 \%$.

The model including $\mathrm{CL} / \mathrm{F}$ and $\mathrm{V} / \mathrm{F}$ scaled to individual body weight and the effect of concomitant prednisolone administration adequately described the CsA concentrations in time as shown by the results of the Visual Predictive Check (Fig. 2), which displays the observed and predicted variability in the concentration measurements. The medians of the observed and simulated CsA concentrations are similar (Fig. 2); the same holds for the $80 \%$ prediction interval compared with the 10th and 90th percentiles of the observed data. This model was therefore considered the base model for further covariate analysis.

Besides body weight and prednisolone use in a daily dose of $20 \mathrm{mg}$ or higher, none of the selected demographic, clinical chemistry or other non-genetic covariates displayed a significant relationship with apparent clearance, apparent central volume of distribution or absorption rate $\left(\mathrm{k}_{\mathrm{a}}\right)$. In addition, none of the selected genetic polymorphisms had a significant relationship with $\mathrm{CL} / \mathrm{F}$ when tested on the base model. Furthermore, no relationship between haplotypes for ABCB1 genotypes and apparent clearance was found. In Table 5 the haplotype combinations and frequencies are presented.

Finally, the AUC monitoring strategy over time in this population of kidney transplant patients is depicted in Fig. 3. This illustrates the procedure of adjusting the daily dose to a preset target in terms of $\mathrm{AUC}_{0-24} \mathrm{~h}$.

\section{Discussion}

Explaining variability in CsA pharmacokinetics is important to reach target exposure early after transplantation. The current trend toward minimizing exposure to calcineurin inhibitors [1] requires insight into the sources of variability in CsA pharmacokinetics, because of an increased risk of acute rejection episodes. A multitude of factors can be responsible for the variability in the pharmacokinetics. From the literature, an array of non-genetic biological and lifestyle-related factors were selected, including age, body size, gender, food intake [3], serum albumin concentration, hematocrit and lipoproteins (HDL, LDL) [6, 7], and coadministration of interacting drugs [3, 8-11] known to affect CsA PK. Moreover, a number of genetic variants in genes encoding for the involved drug-metabolizing enzymes CYP3A4 and CYP3A5 and the multidrug resis- 
Table 4 Population pharmacokinetic parameters for ciclosporin A (CsA) obtained from the bootstrap of the final model. This table shows the mean and coefficient of variation of the pharmacokinetics (PK) parameter estimates as well as the median and percentiles of these estimates. The variability concerns the actual random variability in the PK parameter relative to the population mean value

\begin{tabular}{|c|c|c|c|c|c|}
\hline PK parameter & Mean value & Variability $(\%)$ & CV $(\%)$ & Median & Percentiles $2.5-97.5(\%)$ \\
\hline Absorption rate constant $\left(\mathrm{k}_{\mathrm{a}}, \mathrm{h}^{-1}\right)^{\mathrm{a}}$ & 2.0 & & 11 & 2.0 & 1.6 to 2.5 \\
\hline $\mathrm{DDPR} \geq 20 \mathrm{mg}$ & $-55 \%{ }^{\mathrm{b}}$ & & -10 & $-56 \%$ & -66 to 42 \\
\hline Number of transit compartments & 1 & & & & \\
\hline Transit time or lag time $(\mathrm{h})^{\mathrm{a}}$ & 1 & & & & \\
\hline CsA clearance $(\mathrm{L} / \mathrm{h})$ & 15 & & 4 & 15 & 14 to 16 \\
\hline Central volume of distribution $\left(\mathrm{V}_{\mathrm{c}}\right)(\mathrm{L})$ & 56 & & 7 & 57 & 49 to 64 \\
\hline Peripheral volume of distribution $\left(\mathrm{V}_{\mathrm{p}}\right)(\mathrm{L})$ & 125 & & 10 & 125 & 100 to 149 \\
\hline Intercompartmental clearance $(\mathrm{Q})(\mathrm{L} / \mathrm{h})$ & 14 & & 9 & 14 & 12 to 16 \\
\hline Bioavailability $(\mathrm{F})$ : & 0.5 & & & & \\
\hline $\mathrm{DDPR} \geq 20 \mathrm{mg}$ & $-22 \%^{\mathrm{b}}$ & & -13 & $-22 \%$ & -27 to 16 \\
\hline IIV absorption rate & 0.09 & 30 & 31 & 0.09 & 0.04 to 0.16 \\
\hline IIV clearance & 0.03 & 17 & 24 & 0.03 & 0.02 to 0.05 \\
\hline IIV central volume of distribution & 0.12 & 35 & 40 & 0.11 & 0.05 to 0.24 \\
\hline IOV bioavailability & 0.02 & 14 & 17 & 0.02 & 0.01 to 0.02 \\
\hline Residual variability & 0.07 & 26 & 10 & 0.07 & 0.06 to 0.09 \\
\hline
\end{tabular}

$D D P R$ daily dose prednisolone, $S E$ standard error, $C V$ coefficient of variation, $I O V$ inter-occasion variability; $I I V$ inter-individual variability

${ }^{\text {a }}$ Transit time with one transit compartment is equal to: $1 / \mathrm{k}_{\mathrm{a}} * 2$

${ }^{\mathrm{b}}$ These numbers mean a $55 \%$ lower value for the absorption rate constant and a $22 \%$ lower value for CsA bioavailability

tance transporter ABCB1 [13, 17] were selected. Studies associating single genetic variants in these genes, not considering other non-genetic factors, have been performed, but show conflicting results $[22,30]$. With respect to PXR, there are no studies published that explore the relationship of genetic variants in this gene with CsA PK parameters in adult renal transplant recipients.

This leads to an integrated or population analysis combining genetic and non-genetic factors. A total of seven genetic polymorphisms in $C Y P 3 A 4, C Y P 3 A 5$, and $A B C B 1$ enzymes
Fig. 2 The visual predictive check with the $80 \%$ prediction interval (area between the outer solid lines). The middle solid line represents the median of the model prediction. The observed concentrations are shown as closed symbols, whereas the median of the observed concentrations per time point are shown with the stripe (-) symbol. The dotted lines indicate the 10th and 90th percentiles of the observed data

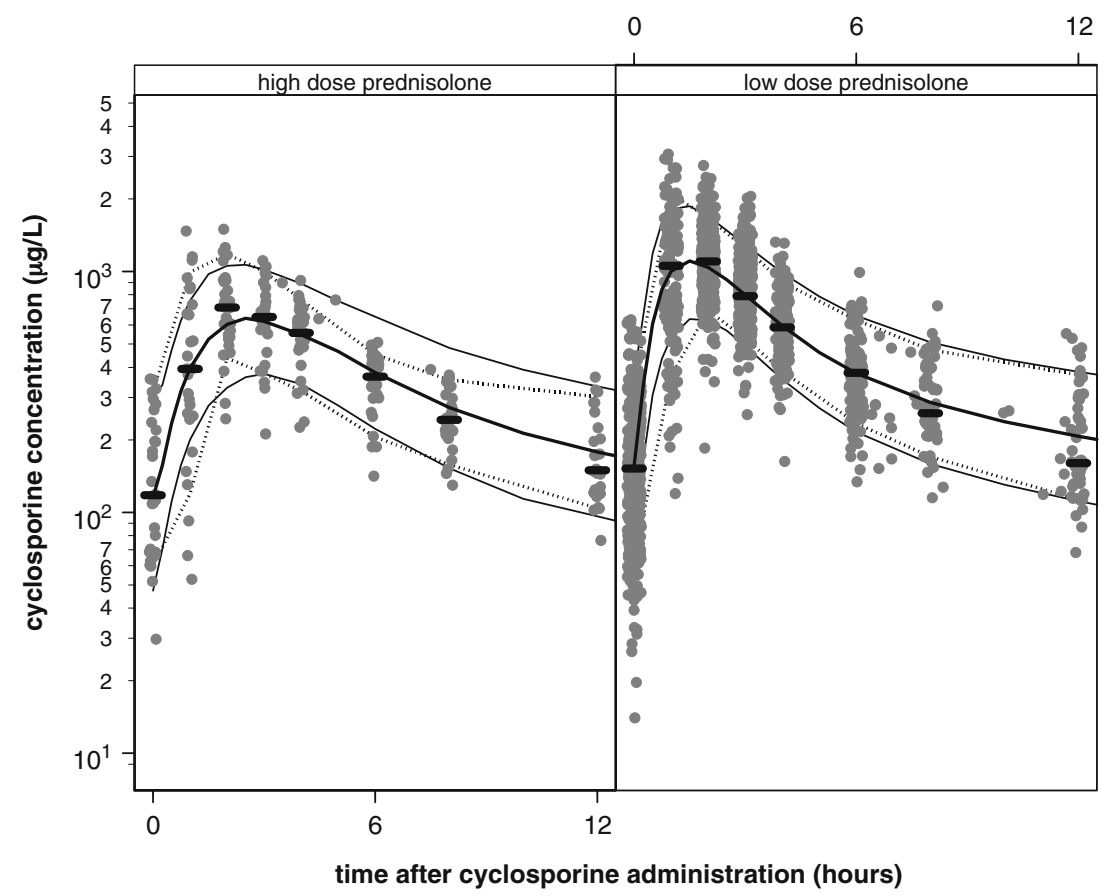


Table 5 ABCB1 haplotype table, which contains respectively the SNPs ABCB1 T3435C (rs1045642), C1236T (rs1128503), and G2677T (rs2032582). This table shows the frequencies of the haplotype combinations on the left side, while on the right side the frequency of the individual triplets among the total amount of 60 triplets, 2 loci (of 3 alleles) $\times 30$ individuals, is presented

\begin{tabular}{lllll}
\hline $\begin{array}{l}\text { Haplotype } \\
(n=30)\end{array}$ & & & \multicolumn{2}{c}{ Total triplets $(n=60)$} \\
\hline HAP1 & HAP2 & $\begin{array}{c}n \text { per } \\
\text { group }\end{array}$ & $\begin{array}{c}\text { Haplotype frequency } \\
(\%)\end{array}$ \\
TTT & CCG & 10 & CCG & 0.45 \\
CCG & CCG & 7 & TTT & 0.38 \\
TTT & TTT & 6 & TCG & 0.10 \\
TTT & TCG & 2 & CTT & 0.03 \\
CTT & CCG & 1 & CTG & 0.03 \\
CCG & CTG & 1 & CCT & 0.01 \\
CTT & TTT & 1 & & \\
TCG & TCG & 1 & & \\
CCG & TCG & 1 & & \\
\end{tabular}

were included in the analysis. In addition, a relationship between the five selected genetic polymorphisms in the pregnane $\mathrm{X}$ receptor could not be related to CsA PK. Interestingly, two covariates did appear to be relevant for individualizing therapy, body weight, and prednisolone dose. In our analysis body weight explained $35 \%$ of the variability in CsA clearance between patients, while a prednisolone dose of over $20 \mathrm{mg}$ /day explained $20 \%$ of the within-patient variability in apparent clearance. After taking the relevant covariates into account, $17 \%$ of inter-individual variability in clearance remained unexplained (Table 4).
Earlier studies have been inconclusive with regard to the relationship between CsA exposure and genetic polymorphisms in CYP3A4 and P-glycoprotein (ABCB1/MDR1) [18-21, 31]. In contrast to the current study, these studies mostly used dose-adjusted trough concentrations as a measure of drug exposure. It is known that trough concentrations correlate poorly with exposure in terms of AUC [4]. In our study full PK profiles were obtained, which made it possible to accurately estimate AUC and hence apparent clearance. In addition, the PK parameter clearance obtained with a population model is very sensitive to detecting a covariate effect (i.e., a genotype effect) as one can account for the contributions of other covariates in the analysis. The population analysis methodology used in the present study differentiates between structural variability (within an individual) and random variability (between individuals). In contrast to nonpopulation-based approaches this results in greater statistical power to identify a covariate effect, because two sources of information are used instead of one. When analyzing multiple observations per subject one is able to compensate for the small number of individuals. Still, this study was not designed primarily to identify genotype effects. Therefore, we performed a posterior power calculation to estimate the minimum genotype effect that could be identified with a power of $80 \%$ and $95 \%$ confidence [32]. This was done for genotypes with a frequency of 10,20 , and $30 \%$ based on Table 3. For a genotype frequency of 10,20 or $30 \%$ the minimum genotype effect that could be detected with a power of $80 \%$ was $55 \%, 35 \%$ or $32 \%$ respectively. This raises the question whether the genotype effects that could not be identified with a power of $80 \%$ (e.g., the effect of
Fig. 3 The $\mathrm{AUC}_{0-24 \mathrm{~h}}$ vs time post-transplantation for every patient included in the analysis. Target exposure is represented by the dotted lines. After week 6, target exposure of ciclosporin A was minimized from $\mathrm{AUC}_{0-24 \mathrm{~h}}$ 10,800 to $6,500 \mu \mathrm{g} * \mathrm{~h} / \mathrm{L}$. Week 2 should be regarded as a grouping variable with the first AUC measurement as early as 5 days post-transplantation and a median of 9 days post-transplantation. $A U C$ area under the blood concentration vs time curve

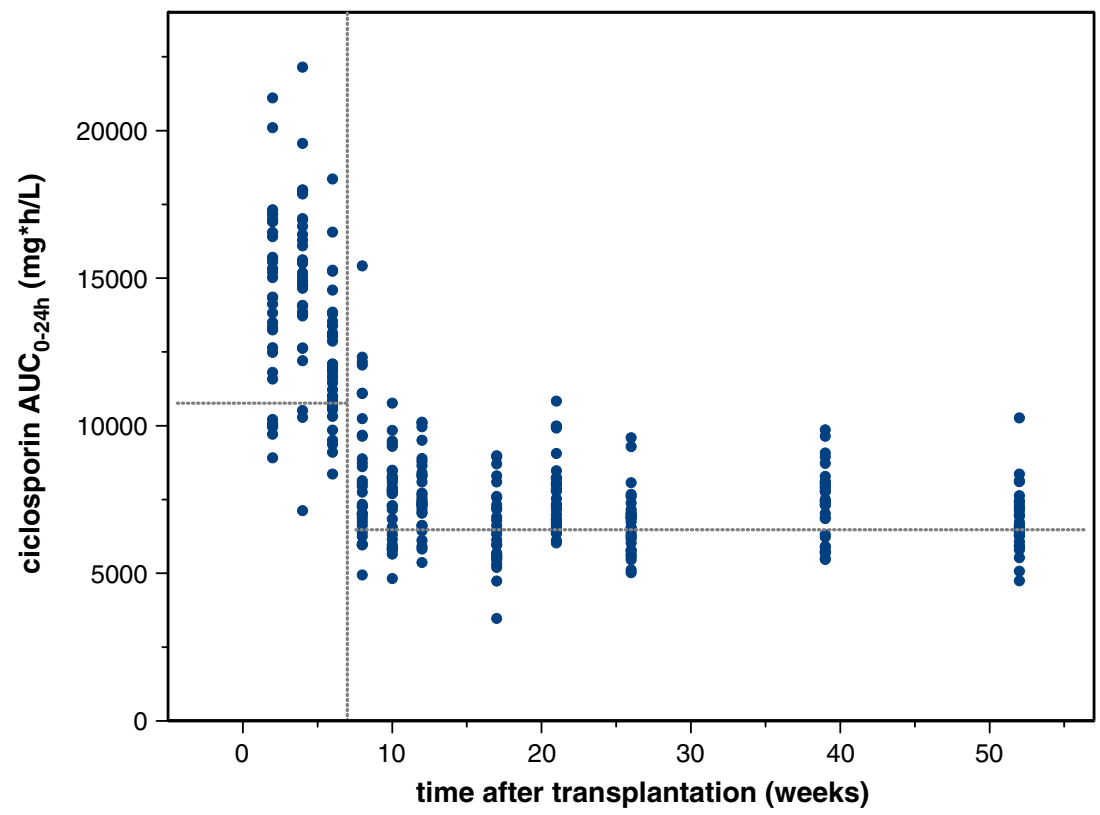


$30 \%$ with a genotype frequency of $20 \%$ ) would be clinically relevant early after transplantation. Therefore, one should consider the dose reduction necessary to reach the target AUC, in this example within 6 weeks after transplantation. The median starting dose was $300 \mathrm{mg}$, while a median dose decrease of $58 \%$ or $125 \mathrm{mg}$ CsA b.i.d. was necessary to obtain a median AUC decrease of around $2,000 \mu \mathrm{g} * \mathrm{~h} / \mathrm{L}$. This demonstrates that relatively large dose steps are clinically necessary early post-transplantation, while the genotype effects appear to be small. In addition, small genotype effects will display overlapping distributions, harming the specificity of this approach when applying clinically.

In the literature, only one study was found that was set up using comparable design and data analysis; in this study, an age-related effect of $A B C B 1$ polymorphisms on CsA oral bioavailability was found [33]. This study was performed in 104 pediatric dialysis patients who received a single pretransplant intravenous CsA dose and a subgroup also received an oral dose at least 1 day later. This design has the advantage that CsA bioavailability could be estimated, since intravenous and oral data were analyzed simultaneously. Both this study and the present study were comparable with regard to the amount of data analyzed as well as inclusion of body weight into the model. The discrepancy with regard to the role of $A B C B 1$ genetic polymorphisms may be explained by differences in study populations and co-medication. Fanta et al. [33] analyzed data from pediatric pre-transplant patients on dialysis who received a single CsA dose without co-administration of prednisolone. Specifically, dialysis patients have typical clinical characteristics such as the presence of a uremic intestine, which could have a marked effect on absorption and perhaps on the activity of the intestinal enzymes. Indeed, adult pre-transplant dialysis patients display highly variable oral bioavailability [34]. Moreover, co-administration of the enzyme inducer prednisolone could potentially mask a relationship between $A B C B 1$ and CsA clearance. Finally, the population presented in our study is a reflection of a typical clinical transplantation cohort with patients for whom therapy with the immunosuppressant CsA is applied. Thus, from our study we can conclude that the model we presented including body weight and prednisolone dose, but without $(A B C B 1)$ genotype is adequate for application in an adult kidney transplant population on triple therapy including prednisolone, within the first year after transplantation.

The SNPs in the gene encoding the pregnane $\mathrm{X}$ receptor (PXR) were not found to be relevant for explaining variability in CsA PK. We hypothesize that the nuclear receptor could be responsible for increased CsA clearance because of induction of $C Y P 3 A 4$ and $A B C B 1$ [35, 36]. Indeed, prednisolone is able to activate $P X R$, as described previously [14], and could in theory be responsible for increased CsA clearance as observed early after transplantation. Inter-individual variability in this drug-drug interaction could potentially be related to polymorphisms in the gene coding for PXR, as hypothesized previously for tacrolimus [37]. Yet, the present analysis revealed that all patients were affected in the same manner by a decrease in CsA apparent clearance early after transplantation, which limited the possibility of finding a relationship between $P X R$ genotype and CsA clearance. Therefore, the covariate prednisolone dose over $20 \mathrm{mg}$ /day appeared to be sufficient to explain the decrease in apparent CsA clearance in time. In addition, the prednisolone dose was rapidly tapered from $100 \mathrm{mg}$ on the day of transplantation to $10 \mathrm{mg}$ once daily in the first 3 weeks post-transplantation and therefore was correlated with time post-transplantation. Specifically, in the first 2 weeks after transplantation a significantly lower bioavailability was estimated. Furthermore, this could also be the result of the improved health status of the patients shortly after transplantation attended with an increase in CsA binding factors, such as serum albumin concentration and hematocrit. However, this analysis did not reveal a covariate effect of these markers. Although unambiguous evidence for the interaction between prednisolone and CsA is lacking [38-40], the effect on bioavailability was attributed to a prednisolone dose in this study and not to time post-transplantation. Yet, to draw conclusions on this matter, CsA PK in the absence of prednisolone should be compared. The effect of prednisolone, or the time posttransplantation effect, on CsA exposure hinders TDM, as can be seen from Fig. 3. After the first AUC visit grouped as week 2, the CsA dose was reduced in the majority of patients. Interestingly, this dose reduction did not result in a lower CsA AUC at the next visit. Because the prednisolone dose was tapered at the same time, CsA clearance decreased and the AUC remained at the same level. Hence, one should account for co-administration of prednisolone when applying a TDM strategy and adjusting the dose.

As described in the Materials and methods section, steady-state PK was assumed for the analysis of these data. Three factors could obscure this assumption, namely dietary fluctuations, variability in dosing intervals, and compliance issues. Patients were allowed to take a light breakfast just prior to their TDM visit in the outpatient clinic. However, most likely the evening before these patients had taken a heavy meal. This dietary variability could cause altered CsA concentrations. The same holds true for daily fluctuations in dosing interval or accidentally missing a dose. These factors cause fluctuations in PK parameters over time and were accounted for with intraoccasion variability.

This population analysis demonstrates that body weight is an important covariate, while the selected genetic polymorphisms appear to have, if any, only a non-clinically 
relevant effect on CsA exposure. In contrast, for tacrolimus genetic factors were relevant for individualized dosing, whereas body weight appeared irrelevant [37]. The two calcineurin inhibitors are often bracketed together because of their grouping and interchangeable use, which suggest that they are comparable drugs. However, one should be careful when comparing these two calcineurin inhibitors as they are chemically and thus pharmacokinetically very different. Both display characteristic absorption profiles, since CsA is mainly absorbed in the upper intestine (duodenum and proximal jejunum) [41], while tacrolimus is absorbed throughout a larger part of the intestine (duodenum down to the ileum) [42]. In addition, these drugs distribute differently throughout the body and both bind to different immunophilins, FK-BP and cyclophilin [43]. In blood both drugs bind to red blood cells, albumin, and $\alpha$-acid protein, but in fairly different ways and to different extents $[6,44,45]$. Finally, they are both metabolized in a unique pattern $[3,46]$.

Both CsA and tacrolimus are subject to dose reduction protocols [1], which inevitably makes variability in exposure an issue for both drugs. CsA displays relatively low inter-individual variability, at least compared with tacrolimus, in which genetic markers have been shown to be relevant for individualized dosing [18, 37]. In clinical practice the variability of CsA is handled with TDM. The unexplained inter-occasion variability (14\%) in apparent clearance is lower than the unexplained inter-individual variability $(17 \%)$, which supports the role of TDM. The low intra-individual variability post-transplantation while on a stable prednisolone dose indicates that as soon as the patients' blood concentrations are adjusted to the target level, the frequency in monitoring visits could be reduced. From that moment onward, monitoring is necessary mostly during conditions, such as infection and diarrhea, and at times when potentially interactive co-medication is started.

\section{Conclusion}

Individualizing CsA treatment in adult kidney transplant recipients can be achieved by a body weight-based dosage followed by a TDM strategy. The CsA dosage should be adjusted to the decrease in apparent CsA clearance in the first weeks after transplantation, possibly as a result of tapering the concomitant prednisolone dose. It appears that the selected genetic polymorphisms in CYP3A4, $C Y P 3 A 5, A B C B 1$, and $P X R$ explain variability in CsA exposure insufficiently to be of clinical relevance. Genotyping for these polymorphisms will probably not lead to an improved dosing strategy for optimizing exposure early after transplantation.
Open Access This article is distributed under the terms of the Creative Commons Attribution Noncommercial License which permits any noncommercial use, distribution, and reproduction in any medium, provided the original author(s) and source are credited.

\section{References}

1. Ekberg H, Tedesco-Silva H, Demirbas A et al (2007) Reduced exposure to calcineurin inhibitors in renal transplantation. N Engl J Med 357:2562-2575

2. Mahalati K, Belitsky P, Sketris I, West K, Panek R (1999) Neoral monitoring by simplified sparse sampling area under the concentration-time curve: its relationship to acute rejection and cyclosporine nephrotoxicity early after kidney transplantation. Transplantation 68:55-62

3. McMillan MA (1989) Clinical pharmacokinetics of cyclosporin. Pharmacol Ther 42:135-156

4. Cremers SC, Scholten EM, Schoemaker RC, Lentjes EG, Vermeij P, Paul LC, den Hartigh J, de Fijter JW (2003) A compartmental pharmacokinetic model of cyclosporin and its predictive performance after Bayesian estimation in kidney and simultaneous pancreas-kidney transplant recipients. Nephrol Dial Transplant 18:1201-1208

5. Knight SR, Morris PJ (2007) The clinical benefits of cyclosporine C2-level monitoring: a systematic review. Transplantation 83:15251535

6. Akhlaghi F, Trull AK (2002) Distribution of cyclosporin in organ transplant recipients. Clin Pharmacokinet 41:615-637

7. Wasan KM, Pritchard PH, Ramaswamy M, Wong W, Donnachie EM, Brunner LJ (1997) Differences in lipoprotein lipid concentration and composition modify the plasma distribution of cyclosporine. Pharm Res 14:1613-1620

8. Rosenbaum SE, Baheti G, Trull AK, Akhlaghi F (2005) Population pharmacokinetics of cyclosporine in cardiopulmonary transplant recipients. Ther Drug Monit 27:116-122

9. Ubhi CS, Woodhouse L, Giles GR (1990) Interaction of intravenous methylprednisolone with oral cyclosporin. Nephrol Dial Transplant 5:376-378

10. Ducharme MP, Warbasse LH, Edwards DJ (1995) Disposition of intravenous and oral cyclosporine after administration with grapefruit juice. Clin Pharmacol Ther 57:485-491

11. Hebert MF, Roberts JP, Prueksaritanont T, Benet LZ (1992) Bioavailability of cyclosporine with concomitant rifampin administration is markedly less than predicted by hepatic enzyme induction. Clin Pharmacol Ther 52:453-457

12. Lown KS, Mayo RR, Leichtman AB, Hsiao HL, Turgeon DK, Schmiedlin-Ren P, Brown MB, Guo W, Rossi SJ, Benet LZ, Watkins PB (1997) Role of intestinal P-glycoprotein (mdr1) in interpatient variation in the oral bioavailability of cyclosporine. Clin Pharmacol Ther 62:248-260

13. Lemahieu WP, Maes BD, Verbeke K, Vanrenterghem Y (2004) CYP3A4 and P-glycoprotein activity in healthy controls and transplant patients on cyclosporin vs. tacrolimus vs. sirolimus. Am J Transplant 4:1514-1522

14. Lamba J, Lamba V, Schuetz E (2005) Genetic variants of PXR (NR1I2) and CAR (NR1I3) and their implications in drug metabolism and pharmacogenetics. Curr Drug Metab 6:369-383

15. Zhang J, Kuehl P, Green ED et al (2001) The human pregnane X receptor: genomic structure and identification and functional characterization of natural allelic variants. Pharmacogenetics 11:555-572

16. Urquhart BL, Tirona RG, Kim RB (2007) Nuclear receptors and the regulation of drug-metabolizing enzymes and drug trans- 
porters: implications for interindividual variability in response to drugs. J Clin Pharmacol 47:566-578

17. Wojnowski L (2004) Genetics of the variable expression of CYP3A in humans. Ther Drug Monit 26:192-199

18. Hesselink DA, van Schaik RH, van der Heiden IP, van der Werf M, Gregoor PJ, Lindemans J, Weimar W, van Gelder T (2003) Genetic polymorphisms of the CYP3A4, CYP3A5, and MDR-1 genes and pharmacokinetics of the calcineurin inhibitors cyclosporine and tacrolimus. Clin Pharmacol Ther 74:245-254

19. Anglicheau D, Thervet E, Etienne I et al (2004) CYP3A5 and MDR1 genetic polymorphisms and cyclosporine pharmacokinetics after renal transplantation. Clin Pharmacol Ther 75:422-433

20. Anglicheau D, Legendre C, Thervet E (2004) Pharmacogenetics in solid organ transplantation: present knowledge and future perspectives. Transplantation 78:311-315

21. Haufroid V, Mourad M, Van Kerckhove V, Wawrzyniak J, De Meyer M, Eddour DC, Malaise J, Lison D, Squifflet JP, Wallemacq P (2004) The effect of CYP3A5 and MDR1 (ABCB1) polymorphisms on cyclosporine and tacrolimus dose requirements and trough blood levels in stable renal transplant patients. Pharmacogenetics 14:147-154

22. Hesselink DA, van Gelder T, van Schaik RH (2005) The pharmacogenetics of calcineurin inhibitors: one step closer toward individualized immunosuppression? Pharmacogenomics 6:323337

23. Karlsson MO, Lindberg-Freijs A (1990) Comparison of methods to calculate cyclosporine A bioavailability from consecutive oral and intravenous doses. J Pharmacokinet Biopharm 18:293-311

24. Anderson BJ, Holford NH (2008) Mechanism-based concepts of size and maturity in pharmacokinetics. Annu Rev Pharmacol Toxicol 48:303-332

25. Wahlby U, Jonsson EN, Karlsson MO (2002) Comparison of stepwise covariate model building strategies in population pharmacokinetic-pharmacodynamic analysis. AAPS PharmSci 4: E27

26. Beal SL, Sheiner LB, Boeckmann AJ (2006) NONMEM users guide (1989-2006). Icon Development Solutions, Ellicott City, MD

27. Wahlby U, Jonsson EN, Karlsson MO (2001) Assessment of actual significance levels for covariate effects in NONMEM. $J$ Pharmacokinet Pharmacodyn 28:231-252

28. Post TM, Freijer JI, Ploeger BA, Danhof M (2008) Extensions to the visual predictive check to facilitate model performance evaluation. J Pharmacokinet Pharmacodyn 35:185-202

29. Bonate PL (2006) Pharmacokinetic-pharmacodynamic modeling and simulation. Springer, New York, pp 355-364

30. Thervet E, Legendre C, Beaune P, Anglicheau D (2005) Cytochrome P450 3A polymorphisms and immunosuppressive drugs. Pharmacogenomics 6:37-47

31. Szekeres T, Haushofer A (2005) Clinical pharmacogenetics of immunosuppressive drugs in organ transplantation. Pharmacogenomics 6:163-168

32. Bertrand J, Comets E, Laffont CM, Chenel M, Mentre F (2009) Pharmacogenetics and population pharmacokinetics: impact of the design on three tests using the SAEM algorithm. J Pharmacokinet Pharmacodyn 36:317-339
33. Fanta S, Niemi M, Jonsson S, Karlsson MO, Holmberg C, Neuvonen PJ, Hoppu K, Backman JT (2008) Pharmacogenetics of cyclosporine in children suggests an age-dependent influence of ABCB1 polymorphisms. Pharmacogenet Genomics 18:77-90

34. Aweeka FT, Tomlanovich SJ, Prueksaritanont T, Gupta SK, Benet LZ (1994) Pharmacokinetics of orally and intravenously administered cyclosporine in pre-kidney transplant patients. J Clin Pharmacol 34:60-67

35. Pascussi JM, Drocourt L, Fabre JM, Maurel P, Vilarem MJ (2000) Dexamethasone induces pregnane $\mathrm{X}$ receptor and retinoid $\mathrm{X}$ receptor-alpha expression in human hepatocytes: synergistic increase of CYP3A4 induction by pregnane $\mathrm{X}$ receptor activators. Mol Pharmacol 58:361-372

36. Pascussi JM, Drocourt L, Gerbal-Chaloin S, Fabre JM, Maurel P, Vilarem MJ (2001) Dual effect of dexamethasone on CYP3A4 gene expression in human hepatocytes. Sequential role of glucocorticoid receptor and pregnane X receptor. Eur J Biochem 268:6346-6358

37. Press RR, Ploeger BA, den Hartigh J, van der Straaten T, van Pelt J, Danhof M, de Fijter JW, Guchelaar HJ (2009) Explaining variability in tacrolimus pharmacokinetics to optimize early exposure in adult kidney transplant recipients. Ther Drug Monit $31: 187-197$

38. Lam S, Partovi N, Ting LS, Ensom MH (2008) Corticosteroid interactions with cyclosporine, tacrolimus, mycophenolate, and sirolimus: fact or fiction? Ann Pharmacother 42:1037-1047

39. Konishi H, Sumi M, Shibata N, Takada K, Minouchi T, Yamaji A (2004) Decrease in oral bioavailability of ciclosporin by intravenous pulse of methylprednisolone succinate in rats. J Pharm Pharmacol 56:1259-1266

40. Konishi H, Sumi M, Shibata N, Takada K, Minouchi T, Yamaji A (2004) Influence of intravenous methylprednisolone pulse treatment on the disposition of ciclosporin and hepatic CYP3A activity in rats. J Pharm Pharmacol 56:477-483

41. Drewe J, Beglinger C, Kissel T (1992) The absorption site of cyclosporin in the human gastrointestinal tract. $\mathrm{Br} \mathrm{J}$ Clin Pharmacol 33:39-43

42. Lampen A, Christians U, Gonschior AK, Bader A, Hackbarth I, von Engelhardt W, Sewing KF (1996) Metabolism of the macrolide immunosuppressant, tacrolimus, by the pig gut mucosa in the Ussing chamber. Br J Pharmacol 117:1730-1734

43. Liu J, Farmer JD Jr, Lane WS, Friedman J, Weissman I, Schreiber SL (1991) Calcineurin is a common target of cyclophilincyclosporin A and FKBP-FK506 complexes. Cell 66:807-815

44. Zahir H, McCaughan G, Gleeson M, Nand RA, McLachlan AJ (2004) Changes in tacrolimus distribution in blood and plasma protein binding following liver transplantation. Ther Drug Monit 26:506-515

45. Zahir H, McCaughan G, Gleeson M, Nand RA, McLachlan AJ (2004) Factors affecting variability in distribution of tacrolimus in liver transplant recipients. Br J Clin Pharmacol 57:298-309

46. Venkataramanan R, Swaminathan A, Prasad T, Jain A, Zuckerman S, Warty V, McMichael J, Lever J, Burckart G, Starzl T (1995) Clinical pharmacokinetics of tacrolimus. Clin Pharmacokinet 29:404-430 